\title{
Event Shape Studies at LEP
}

\section{Daniel Wicke}

CERN, CH-1211 Genève 23, Switzerland

E-mail: Daniel. Wicke@cern.ch

ABSTRACT: Infrared and collinear events shapes are suited to directly probe properties of hard QCD. They are traditionally used to measure the strong coupling and to test the gauge structure of QCD. Perturbative predictions exist in several variations all of which depend on the renormalisation scheme leading to large theoretical uncertainties in the determination of $\alpha_{s}$. In order to match perturbative predictions with data the nonperturbative effects of hadronisation have to be taken into account. Beside MC models the analytical power corrections are now widely used.

\section{Introduction}

Event shapes are sensistive to the strong coupling, $\alpha_{s}$, and the gauge structure of the strong force. However, the observables investigated (mean values, higher moments and normalised distributions) don't depend on the production rate nor on the event orientation and are therefore independent of the electroweak production process. They are thus directly connected to fundamental properties of the strong force.

QCD predictions for these observables exist in several approximations. Fixed order $\alpha_{s}$ calculations exist in NLO (in this talk I concentrate on $\mathcal{O}\left(\alpha_{s}^{2}\right)$, see [i] for $\mathcal{O}\left(\alpha_{s}^{3}\right)$ ). NLLA calculations resum all orders of $\alpha_{s}$ in two jet like configurations. Combination of $\mathcal{O}\left(\alpha_{s}^{2}\right)$ with NLLA predictions suffer from ambiguities in avoiding double counting. Several so called matching schemes exist, the most popular being the $\log R$-matching. All of these calculations depend on the unphysical renormalisation scale, which is varied in order to estimate theoretical uncertainties.

The conversion of perturbatively accessible partons into hadrons may have a significant impact on the final value of an event shape observable. In order to match perturbative predictions with data these non-perturbative hadronisation effects thus have to be taken into account. Traditionally the only way to correct for these non-perturbative effects was the application of Monte Carlo models, which suffer from a large number of free parameters that need to be tuned. Since a few years the analytical ansatz of power corrections with only one free parameter is used as an alternative.

I will discuss the main aspects of new results obtained with event shapes and presented

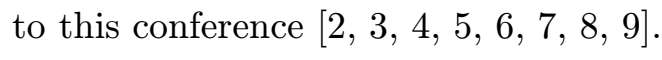




\section{Measurements}

Experimentally the measurement of event shapes observables in $e^{+} e^{-}$is well established. After the detector dependent selection of tracks and clusters the event selection needs to suppress certain backgrounds. At $M_{Z}$ the main backgrounds are Bhabha, $\tau^{+} \tau^{-}$and $\gamma \gamma$ events, which can be efficiently suppressed. At LEP2 on top of these initial state radiation (ISR) and boson ( $W$ or $Z$ ) pair production needs to be taken into account. Especially the suppression of boson pair production is difficult to achieve without biasing the event shape observables. Thus a moderate suppression minimising the bias is combined with subtracting the expected contribution from remaining background. In a final step the measured quantities are corrected for detector acceptance effects to reach results comparable between experiments. However, different experiments use different reference levels in order to minimise systematic errors. As reference levels stable particles including or not including neutrinos and charged particle only are in use. Differences between these levels need to be accounted for when really comparing results.

\section{Interpretation}

\subsection{Strong coupling using MC models}

Based on such measurements all LEP experiments have presented a determination of the strong coupling using combined $\mathcal{O}\left(\alpha_{s}^{2}\right)+$ NLLA in $\log R$-scheme with traditional Monte Carlo

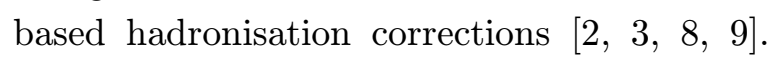
DELPHI and L3 used $1-T, \rho_{h}, C, B_{t}$ and $B_{w}$, ALEPH and OPAL used in addition $y_{23}$. The results are in good agreement with each other. Combination yields

$\alpha_{s}(206 \mathrm{GeV})=0.1082 \pm 0.0012_{\exp } \pm 0.0034_{\text {theo }}$.

Beside combined $\mathcal{O}\left(\alpha_{s}^{2}\right)+$ NLLA DELPHI investigated $\mathcal{O}\left(\alpha_{s}^{2}\right)$ with optimised scales and also pure NLLA and found good agreement within the large theoretical uncertainties.

The LEP QCD working group has performed a combination of direct measurements

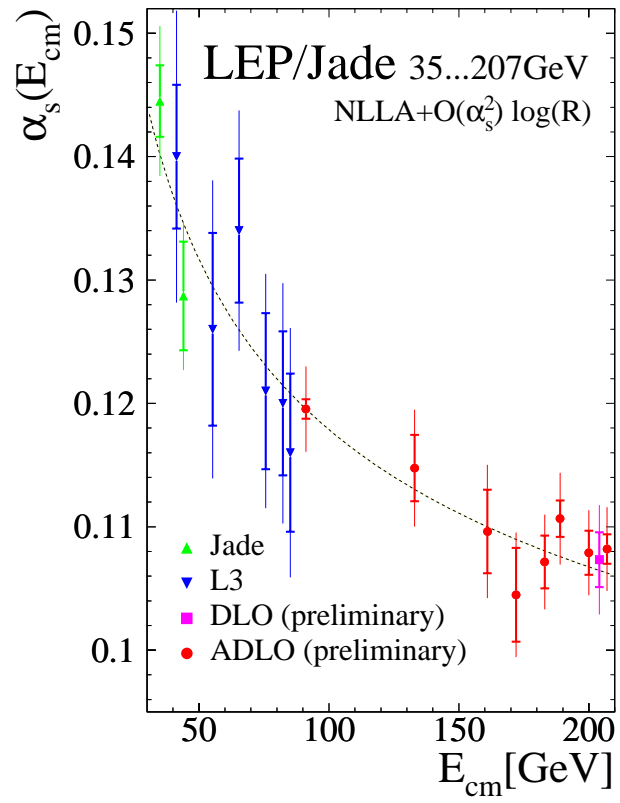

Figure 1: Running of $\alpha_{s}$ for LEP combined results compared to JADE results. Errors are split into a statistical part and the total error. of the strong coupling for centre-of-mass energies between 41 and $205 \mathrm{GeV}$. The inputs collected from existing publications were given by the four LEP experiments with an agreed error split, so that correlations between the experiments and between different energies could be taken into account. An overall $\mathcal{O}\left(\alpha_{s}^{3}\right)$-fit yields

$$
\alpha_{s}\left(M_{Z}\right)=0.1195 \pm 0.0007_{\exp } \pm 0.0048_{\text {theo }}
$$


see line in Fig. 1ir. The result for $206 \mathrm{GeV}$ doesn't enter the fit, yet. The quality of the fit with $\chi^{2} /$ ndf $=31.3 / 36$ shows that the data are in excellent agreement with the running expected from QCD.

An improved combination including the highest energies is in preparation. It will be based on refitted results with an unified theoretical basis and a more homogenous error estimation.

\subsection{Power Corrections}

Power corrections are an analytical description of the influence of hadronisation on event

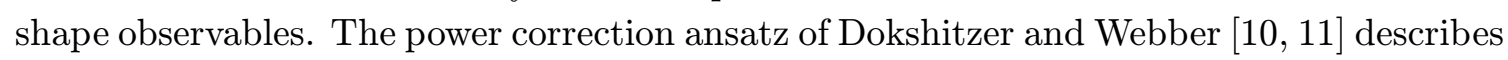
the hadronisation by an additive term for the mean $\langle f\rangle$ of an observable $f$ and by a shift for its distribution $\mathcal{D}_{f}(f)$ :

$$
\langle f\rangle=\left\langle f^{\text {pert }}\right\rangle+c_{f} \mathcal{P}\left(\alpha_{0}\right) \quad \mathcal{D}_{f}(f)=\mathcal{D}_{f}^{\text {pert }}\left(f-c_{f} \mathcal{P}\left(\alpha_{0}\right)\right),
$$

where the superscript 'pert' shall indicate the perturbative prediction. The size of the power correction term is defined by two parts.

All dependence on the variables is put in the coefficient $c_{f}$, which is computed for several observables, see e.g. [i, $\overline{6}$ ] for a table. The other factor, $\mathcal{P}\left(\alpha_{0}\right)$, is the same for all observables. It is falling off like the inverse centre-of-mass energy and depends on an unknown parameter $\alpha_{0} . \alpha_{0}$ is interpreted as the average strong coupling below the intermediate scale $\mu_{I}$ where the perturbative prediction is replaced by the power correction.

An application of power corrections will always have to fit $\alpha_{0}$ from data at low centreof-mass energies, which have the highest sensitivity on this parameter.

\subsubsection{Structure constants}

To measure the structure constants, one needs to use predictions in which the structure constants are still parameters. Second order predictions will have terms proportional to $C_{F}, C_{F}^{2}, C_{F} C_{A}$ and $C_{F} T_{R}=C_{F} \frac{1}{2} n_{\mathrm{f}}$.

In previous measurements MC based hadronisation corrections were used, in which the hadronisation is described assuming of QCD. MC tuning to real data should have minimised the resulting bias.

In [5. for the first time power corrections are used and the structure constants were allowed to vary also in the description of the hadronisation. The combined results of event shape distributions with centre-of-

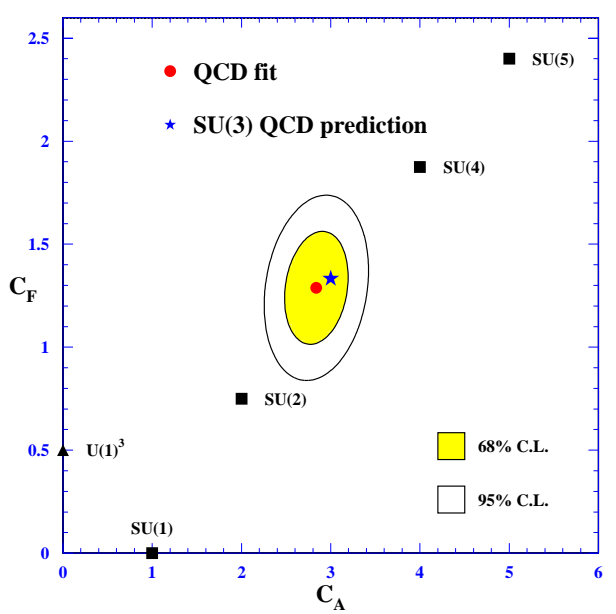

Figure 2: Measurement of structure constants using power corrections [i5in]. mass energies between 14 and $189 \mathrm{GeV}$ obtained for fixed $\alpha_{0}$ are in good agreement with the QCD expectation, as shown in Fig. ${ }_{2}^{2}$ 


\subsubsection{Hadron Masses}

As QCD calculations for both the perturbative and the power corrections were carried out for massless partons, power correction analyses don't take the influence of hadron masses into account. Replacing the fourmomenta of the particles according to

$$
\begin{aligned}
p & =(\vec{p}, E) \rightarrow(\vec{p},|\vec{p}|) \quad(p \text {-scheme }) \\
\text { or } p & =(\vec{p}, E) \rightarrow(\hat{p} E, E)(E \text {-scheme })
\end{aligned}
$$

doesn't change the predictions, but it does change the observable values calculated with massive particles.

For the jet masses at $M_{Z}$ the difference between the usual and the $p$-scheme definition is around $10 \%$, the difference between $E$ - and $p$-scheme $2.5 \%$.

These differences, which demonstrate the influence of hadron masses, behave like a (logarithmically enhanced) power correction. Only in the $E$-scheme is this correction proportional to the same coefficient as the standard power correction: $c_{f}$. Thus performing the power corrections analysis in the $E$-scheme should improve the consistency obtained when fitting $\alpha_{0}$ from different observables.

Fig. 'i3' shows that indeed, when using observables measured in or corrected to the $E$-scheme, the range of $\alpha_{0}$-values reduces from $\pm 25 \%$ to $\pm 12 \%$ [i $\left.6 \overline{1}\right]$.

\subsection{Renormalisation Group Invariant Perturbation Theory}

Renormalisation group invariant perturbation theory (RGI) is based on the idea to use the observable itself as expansion parameter. With this one avoids any dependence on a renormalisation scheme. The energy dependence of an event shapes mean value can still be predicted and is given by the $\beta$-function. The constant from integrating the corresponding relation can be connected to $\alpha_{s}\left(M_{Z}\right)$ using the Celmaster-Gonsalves equation.

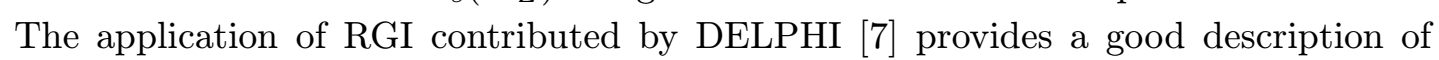
seven observables, when the $E$-scheme definitions is used for the jet masses, and yields $\alpha_{s}\left(M_{Z}\right)=0.1172 \pm 0.0040$. Hadronisation is described by fitting a power correction term, which contributes of the order of only $2 \%$ and is consistent with zero.

For the final result the power correction term is set to zero. This application of pure RGI even reduces the spread on the strong coupling: $\alpha_{s}\left(M_{Z}\right)=0.1195 \pm 0.0020$. For the $\beta$-function a fit including low energy and DELPHI data points yields

$$
\frac{\beta_{0}}{2 \pi}=1.25 \pm 0.05 \quad \text { or, assuming QCD } \quad n_{\mathrm{f}}=4.75 \pm 0.44
$$

with surprisingly small systematics and in perfect agreement with QCD, from which 1.27 is expected. 


\section{Comparison of the various methods}

As in other fields it is useful to compare the direct measurements with the results from

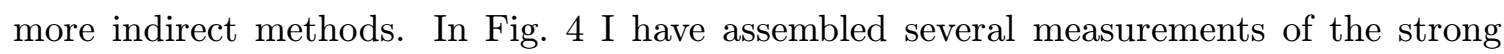
coupling performed at LEP and compared them to the current PDG world average [i]

Beside the results from the LEP QCD working group [i]

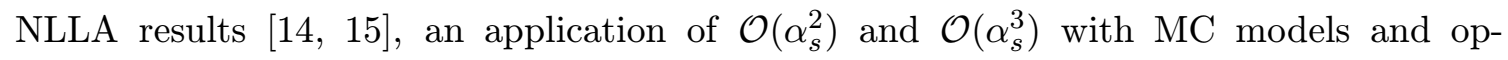

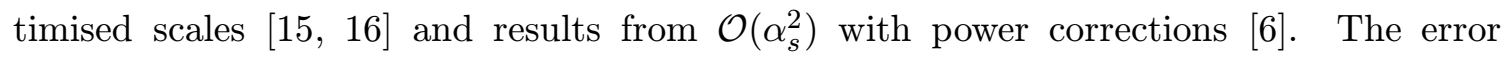
contribution from the renormalisation scale was adapted to use a consistent variation of $\mu / Q=0.5 \ldots 2.0$. The increase, if any, is shown as extra (red) error bars.

The different methods show a good consistency within the quoted errors which are in all cases dominated by the contribution from the renormalisation scale variation.

The indirect determinations of the strong coupling stem from the investigation of $\tau$

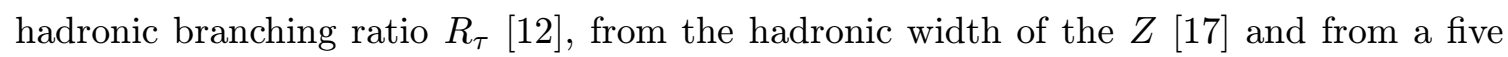
parameter fit to electroweak precision data [i] $\overline{1} \overline{8}]$. They show a good consistency with the direct results and with the world average within the given errors.

\section{Conclusions}

Event shapes provide a means for directly probing properties of hard QCD. There are still many different options on both the perturbative and the non-perturbative side. Experimentalists and theorists are interacting to improve the understanding. The incredibly good description of mean event shapes with pure RGI gives new input to this discussions.

Measurements of the strong coupling from event shapes are in good agreement with results from more indirect measurements. The dependence on the renormalisation scale is used to estimate theoretical uncertainties, which limits the precision of current direct measurements of the strong coupling.

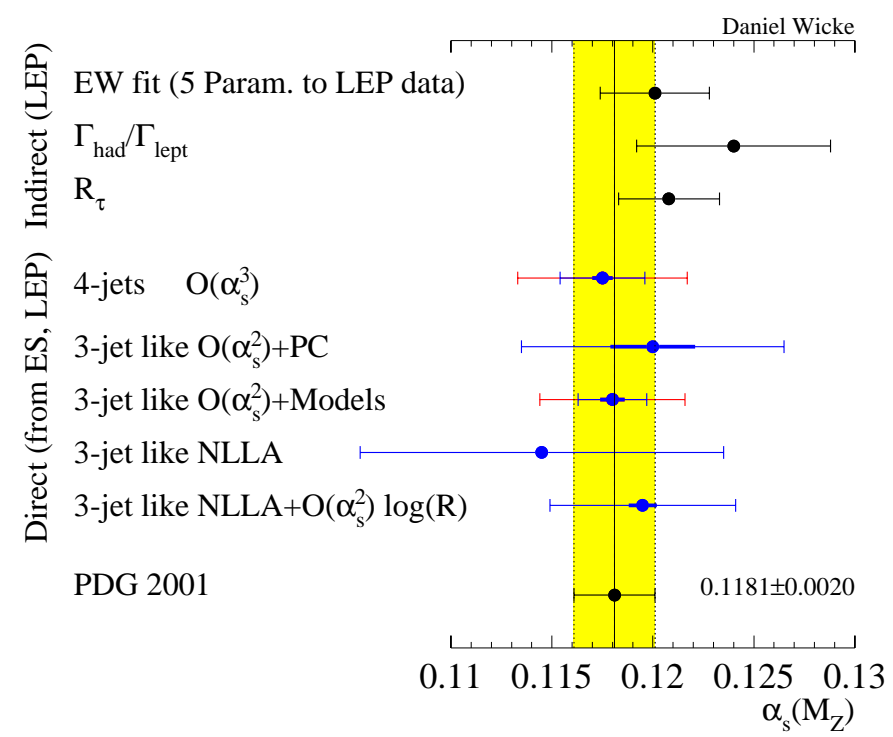

Figure 4: Comparison of direct measurements of the strong coupling from event shapes at LEP with indirect results from LEP and the current world average. 


\section{References}

[1] J. Vossebeld, "Jet \& multiplicity studies at LEP." In these proceedings.

[2] OPAL Collaboration, "Measurement of standard model processes in $e^{+} e^{-}$Collisions at $\sqrt{s} \sim 203-209$ GeV." OPAL Physics Note PN469, EPS \#205, 2001.

[3] ALEPH Collaboration, "Measurements of $\alpha_{s}$ at centre-of-mass energies between 91 and 206 GeV." ALEPH 2001-062 CONF 2001-042, EPS \#212, 2001.

[4] P. A. Movilla Fernández, S. Bethke, O. Biebel, and S. Kluth, "Tests of power corrections for event shapes in $e^{+} e^{-}$annihilation." MPI-Ph/2001-005, EPS \#269, 2001.

[5] S. Kluth, P. A. Movilla Fernández, S. Bethke, C. Pahl, and P. Pfeifenschneider, "A measurement of the QCD colour factors using event shape distributions at $\sqrt{s}=14$ to 189 GeV." MPI-PhE/2001-19, EPS \#271, 2001.

[6] G. P. Salam and D. Wicke, Hadron masses and power corrections to event shapes, JHEP 05 (2001) 061, [arXivinep-ph/0102343']. EPS \#275.

[7] R. Reinhardt, U. Flagmeyer, K. Hamacher, O. Passon, and D. Wicke, "A study of the energy evolution of event shape distributions and their means with the DELPHI detector at LEP." DELPHI 2001-062 CONF 490, EPS \#310, 2001.

[8] O. Passon, J. Drees, U. Flagmeyer, K. Hamacher, R. Reinhardt, and D. Wicke, "QCD resuilts from DELPHI measurements of event shape and inclusive particle distributions at the highest LEP energies." DELPHI 2001-065 CONF 493, EPS \#313, 2001.

[9] L3 Collaboration, "QCD results at $192 \leq \sqrt{s} \leq 208 \mathrm{GeV}$." L3 Note 2670, EPS \#510, 2001.

[10] Y. L. Dokshitzer and B. R. Webber, Calculation of power corrections to hadronic event shapes, Phys. Lett. B352 (1995) 451.

[11] Y. L. Dokshitzer, A. Lucenti, G. Marchesini, and G. P. Salam, Universality of $1 / Q$ corrections to jet-shape observables rescued, Nucl. Phys B511 (1997) 396, [hep-ph/9707532].

[12] D. E. Groom et. al., Review of particle physics, Eur. Phys. J. C15 (2000) 1.

[13] LEP QCD WG, "Preliminary combination of $\alpha_{s}$ values derived from event shape variables at LEP." ALEPH 01-038 Physic 01-012, DELPHI 2001-043 PHYS 893, L3 note 2661, OPAL TN689, 2001.

[14] OPAL Collaboration, R. Akers et. al., A study of QCD structure constants and a measurement of $\alpha_{s}\left(M_{Z^{0}}\right)$ at LEP using event shape observables, Z. Phys. C68 (1995) 519.

[15] DELPHI Collaboration, P. Abreu et. al., Consistent measurements of $\alpha_{s}$ from precise oriented event shape distributions, Eur. Phys. J. C14 (2000) 557-584, [arXiv: hep-ex/0002026i].

[16] U. Flagmeyer, J. Drees, K. Hamacher, O. Passon, R. Reinhardt, and D. Wicke, "Measurement of the strong coupling $\alpha_{s}$ and its energy dependence from the four jet rate of hadronic events with the DELPHI detector at LEP." DELPHI 2001-059 CONF 487, EPS \#307, 2001.

[17] S. Bethke, Determination of the QCD coupling $\alpha_{s}$, J. Phys. G26 (2000) R27, larXivinep-ex/0004021].

[18] The LEP Collaborations, the LEP Electroweak, SLD Heavy Flavour and Electroweak Working Groups, A combination of preliminary electroweak measurements and constraints on the standard model, arXiv: hep-ex/0103048'. CERN-EP/2001-021, updates available on http:/Jern.ch/LEPEWWG/: 